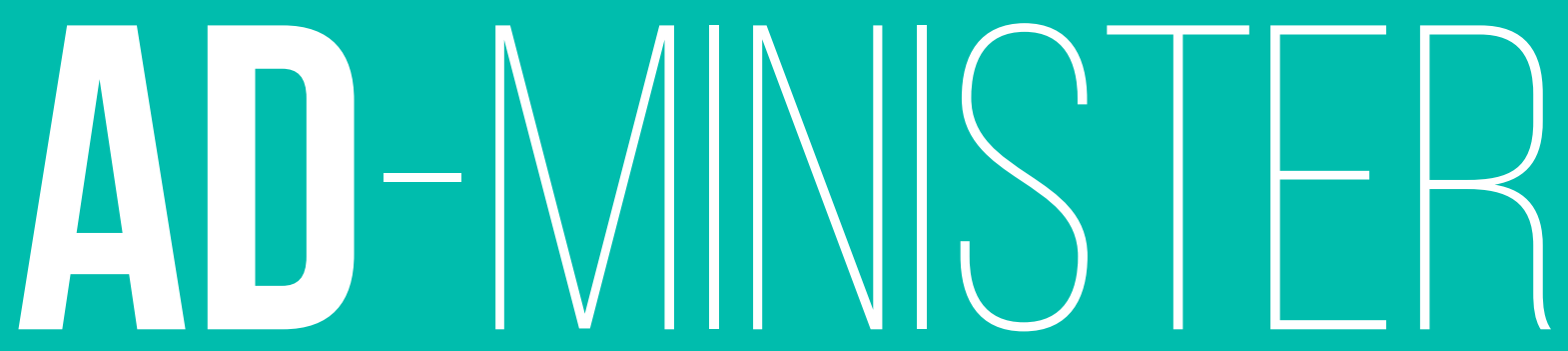

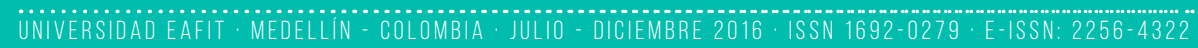

CARLOS EDUARDO

MARULANDA ECHEVERRY

MARCELO

LÓPEZ TRUJILLO

JOSÉ FERNANDO

CASTELLANOS GALEANO

JEL: M150, L8, L86

DOI: $10.17230 /$ ad-minister.29.8 


\title{
LA CULTURA ORGANIZACIONAL Y SU INFLUENCIA EN LAS BUENAS PRÁCTICAS PARA LA GESTIÓN DEL CONOCIMIENTO EN LAS PYMES DE COLOMBIA
}

\author{
ORGANZZATIONAL CLLTURE AND ITS INFLUENCE ON BEST PRACTICES FOR KNOWLEDGE \\ MANAGEMENT IN SMES IN COLOMBIA
}

\section{CARLOS EDUARDO \\ MARULANDA ECHEVERRY ${ }^{1}$ \\ MARCELO \\ LÓPEZ TRUJILLO \\ JOSÉ FERNANDO \\ CASTELLANOS GALEANO ${ }^{3}$}

JEL: M150, L8, L86

RECIBIDO: $29 / 06 / 2016$

MODIFICADO: 31/09/2016

ACEPTAD0: $31 / 10 / 2016$

DOI: $10.17230 /$ ad-minister.29.8 www.eafit.edu.co/ad-minister Creative Commons (CC BY)

\section{RESUMEN}

El objetivo del presente trabajo es la exposición de la relación existente entre la cultura organizacional y las buenas prácticas para la gestión del conocimiento en las Pymes de Colombia, la cual parte de un modelo de evaluación de gestión del conocimiento que fue aplicado a 321 empresas. Para el efecto, se utilizaron métodos de análisis y síntesis y un tipo de estudio descriptivo exploratorio y correlacional. Se concluye que la cultura modera de forma positiva y significativa las buenas prácticas para la gestión de conocimiento. Este estudio se espera que aporte al desarrollo de las pequeñas y medianas empresas del país para formular acciones que permitan potenciar su crecimiento.

\section{PALABRAS CLAVE}

Gestión del conocimiento; cultura organizacional; buenas prácticas para la gestión del conocimiento, pymes.

\section{ABSTRACT}

The aim of this paper is the presentation of the relationship between organizational culture and best practices for knowledge management of SMEs in Colombia, which is part of an evaluation model of knowledge management, which was applied to 321 companies. For the purpose of analysis and synthesis methods and a type of exploratory and correlational descriptive study they were used. It is concluded that culture moderates significantly positive and good practices for knowledge management. This study is expected to contribute to the development of small and medium enterprises in the country to formulate actions to enhance their growth.

\section{KEYWORDS}

Knowledge management; organizational culture; best practices for knowledge management, SMEs.

\footnotetext{
1 Dr. (c). Profesor de la Facultad de Administracion de la Universidad Universidad Nacional de Colombia, sede Manizales y de la Facultad de Ingeniería de la Universidad de Caldas. Manizales, Colombia. Correos electrónicos: cemarulandae@unal.edu.co y carlose@ucaldas.edu.co. ORCID: http:// orcid.org/0000-0002-6287-9386

2 Dr. Profesor de la Facultad de Administracion de la Universidad Universidad Nacional de Colombia, sede Manizales y de la Facultad de Ingeniería de la Universidad de Caldas, Manizales, Colombia. Correos electronicos: mlopeztr@unal.edu.co y mlopez@ucaldas.edu.co. ORCID: http://orcid.org/oooo0003-0668-1292

3 MsC. Profesor asociado de la Facultad de Ingeniería de la Universidad de

Caldas, Manizales, Colombia. Correo electronico: jose@ucaldas.edu.co. ORCID: http://orcid.org/oooo-
} $0002-0743-0359$ 


\section{INTRODUCCIÓN}

La gestión del conocimiento (GC) se ha convertido, en la actualidad, en una de las tendencias más importantes para las organizaciones, reconociendo a los empleados como uno de sus capitales más valiosos y en los cuales reside el conocimiento, producto del desarrollo teórico de los conceptos y de la aplicación en su experiencia relacionada.

Es desde esta gestión, que autores como Wang y Yang (2016) reconocen el potencial para lograr que las Pymes sean más competitivas e innovadoras, que generen un rendimiento sostenible, y que su uso genere un mayor rendimiento individual y grupal, desde sus mejores prácticas.

En este sentido, Alavi y Leidner (2001) señalan que el uso de las mejores prácticas en la GC con frecuencia se apoya en las tecnologías de información y comunicaciones (TIC), conduciendo a resultados positivos en la organización, como la mejor comunicación y mayores niveles de participación entre los miembros del personal, la eficiencia en la resolución de problemas y mejoras en el tiempo de salida de un producto al mercado, el rendimiento financiero más favorable, mejores prácticas de comercialización, mejora en el rendimiento del trabajo en equipo y, por lo tanto, el reconocimiento generalizado de las contribuciones de la GC para el éxito general de una organización.

Esto en el marco de la cultura organizacional, la cual se puede definir como el conjunto de hábitos, valores, prácticas, procedimientos, normas, reglas, comportamientos grupales e individuales de una organización, y que reconocen a esta de manera única e individual. Aporta al desarrollo de la GC en cualquier organización, reconociendo en diversos resultados de investigación, como los de Li y Tsai (2009); Allameha, Zamani y Reza-Davoodia (2011); y Wiewiora, Trigunarsyah, Murphy y Coffey (2013), que, sin duda, es un factor definitivo para una dinámica competitiva propia de las necesidades de hoy.

Los resultados de investigación que se presentan en este artículo parten de la pregunta: ¿cuál es la relación existente entre la cultura organizacional y las buenas prácticas para la gestión del conocimiento de las Pymes de Colombia?, a partir de la cual se plantea una hipótesis relacionada con la influencia de la cultura organizacional en las buenas prácticas para la gestión del conocimiento, dada la importancia de diagnosticar el estado de estas variables en el desarrollo de las Pymes, para así establecer estrategias que permitan su mejoramiento.

\section{GESTIÓN DEL CONOCIMIENTO}

La GC es definida por algunos autores como una disciplina que pretende centrar el desarrollo del conocimiento en fases alrededor de: la adquisición, el almacenamiento, la transformación, la distribución y la aplicación, para obtener ventajas diferenciadoras y competitivas (Barney, 1991; Dosi, Teece, \& Winter, 1991; Riesco, 2006).

También se puede ver como un proceso permanente y continuo que pretende asegurar la generación, el desarrollo y utilización de cualquier conocimiento de va- 
lor, pertinente y adecuado para una organización, cuya pretensión se enfoca a mejorar y aumentar la capacidad de resolver problemas simples y complejos, y ayudar a la sostenibilidad de sus ventajas competitivas (Andreu \& Sieber, 1999).

Durana, Çetindereb y Şahanc (2014) explican que la GC es un proceso para facilitar las actividades relacionadas con el conocimiento, tales como su creación, transformación y uso. El objetivo de la GC es crear una organización de aprendizaje que evalúa, almacena, utiliza y comercializa experiencia y conocimiento auténtico de los empleados de la organización, con el fin de establecer una organización más potente y valiosa, más allá del total de cada componente que la constituye y más eficiente que sus competidores, propiciando ambientes de articulación organizacional interna y externa. Los siguientes puntos se pueden mencionar como utilidad de la GC en las organizaciones:

- Para aumentar el rendimiento, la productividad y la competitividad de la organización

- Para obtener, compartir y utilizar el conocimiento dentro de la organización de manera efectiva

- Para mejorar las decisiones de la organización

- Para mejorar los procesos de negocio y obtener buenas prácticas

- Para reducir los costes de investigación y retrasos

Terzieva (2014) explica que diferentes autores han dado definiciones diversas de GC y, sin embargo, ninguna de ellas es del todo precisa, ni es completamente inexacta. La definición correcta de GC cambia de acuerdo a cada organización, está típicamente ligada a los objetivos de estas y tiene la intención de lograr resultados específicos, los cuales pueden incluir: un mejor rendimiento, la innovación, la ventaja competitiva, las lecciones aprendidas y el desarrollo general de las prácticas de colaboración.

La GC tiene por objeto mejorar el rendimiento mediante el aprovechamiento y mantenimiento presente y futuro del valor de los activos de conocimiento. Se han reconocido la creación, actualización, disponibilidad, calidad y uso del conocimiento como componentes esenciales de una organización de gestión proactiva. Los conceptos clave incluyen la conversión de datos, visión organizativa y experiencia en conocimiento reutilizable y útil que se distribuye y comparte con las personas que lo necesitan. Si una organización gestiona el conocimiento con éxito, será capaz de utilizar la información y consultar a la experiencia, lo cual le será muy útil, incluso en algunos casos críticos, para hacer un mejor trabajo que el que ha sido hecho anteriormente (Collins \& Smith, 2006; Mesmer-Magnus \& DeChurch, 2009).

Hoy día ha aumentado la importancia y la necesidad de una utilización eficiente del conocimiento en las organizaciones. Es un hecho que las personas aprenden de la experiencia, sobre todo si repiten actividades similares, porque comienzan por reconocer las diferentes situaciones y aprender a evitar o hacer frente a otras. La GC 
permite a los miembros de un equipo reducir los reprocesos y ajustar el tiempo que se necesita para planificar la ejecución de un proyecto. Compartir las lecciones aprendidas y prácticas avanzadas, de hecho, se sugiere como una clave para ayudar a las empresas.

\section{CULTURA ORGANIZACIONAL}

Según Durana et al. (2014), la creación de una visión común en relación con el conocimiento y el hecho de compartir valores dentro de la organización serán comportamientos efectivos para el éxito del proceso de GC. La creación de una cultura de intercambio de conocimientos depende del respeto dentro del equipo, la confianza mutua, y las relaciones positivas individuales y de grupo.

La GC se relaciona con generar, almacenar y aplicar el conocimiento, lo que crea valor para la organización. Algunas condiciones tácitas individuales dentro de la empresa, como lo son las experiencias, las ideas, los pronósticos y las prácticas, son difíciles de formular y transmitir. En esencia, el proceso de GC se lleva a cabo mediante la transformación del conocimiento organizacional en otro tipo de saber.

En este sentido, Power y Cormicana (2015) explican que es posible consolidar una organización que tenga una cultura apropiada para promover la creación, transferencia y reutilización del conocimiento. Esto se logra mediante el desarrollo de una cultura de apertura y de compartir, mediante la motivación, la participación de personas y la incorporación de las actividades de GC en el día a día de los procesos de negocio, sistemas y estructuras internas. Las personas centradas en cuestiones tales como la calidad, las interacciones humanas, las motivaciones y los procesos de toma de decisiones tienen un efecto más fuerte en comparación con otros factores, como pueden ser los procesos organizacionales, los sistemas o las estructuras.

En lo relacionado con la cultura, las recompensas y los incentivos demuestran tener una relación positiva en el éxito de un proyecto. Los premios tienen una influencia positiva en la transferencia de conocimiento, mientras que las recompensas son reconocidas como importantes en la motivación de los empleados para realizar tareas específicas dentro de una organización. La falta de recompensas en una organización es un obstáculo importante en la transferencia de conocimiento. Por otro lado, la comunicación tiene una relación positiva con el éxito de un proyecto y es un atributo esencial en la transferencia de conocimientos. Esta transferencia se da también con la comunicación oral y el uso del lenguaje corporal.

Según Allameha, Zamani y Reza-Davoodia (2011), y Lin y Lee (2012), la cultura influye en los comportamientos que tienen relación con la GC, ya que los seres humanos y los grupos formales y no formales en una organización interactúan de tal manera que definen cuándo, dónde y con quién relacionarse e intercambiar y transferir conocimiento, lo que hace que la cultura pueda ser mejorada, cambiada, transformada y se afecten de manera directa los procesos que tiene que ver con la selección de personal, el manejo de los incentivos y las recompensas, el trabajo en equipo, la gestión de ideas y proyectos, la innovación y el aprendizaje, entre otros. 


\section{BUENAS PRÁCTICAS PARA LA GC}

Básicamente, Villar, Alegre y Pla-Barber (2014) consideran que las prácticas de GC son las rutinas organizativas orientadas hacia su explotación, buscando la aplicación de conocimientos para facilitar el desarrollo de las rutinas y capacidades, y difundir conocimientos y prácticas de almacenamiento relevantes en la organización.

Las prácticas de difusión del conocimiento incluyen aquellos procesos que permiten la aplicación de este a través de canales formales e informales, el cual es distribuido tanto dentro como fuera de la firma, así como técnicas para fomentar la motivación de los empleados para compartir el conocimiento y la participación a través de los círculos de calidad o equipos multidisciplinares. La existencia de estructuras de conocimiento es particularmente relevante en las Pymes. De otro lado, las prácticas de almacenamiento de conocimiento son sistemas desarrollados para apoyar los procesos de recuperación del conocimiento operacional y de almacenamiento, e incluyen sistemas de procesamiento de información, procedimientos de control y revisión, y sistemas que permiten el uso del stock de conocimiento creado. Esta estructura habilita la recolección y transformación de la información pertinente y su aplicación para otras operaciones, proporcionando una retroalimentación valiosa.

Donate y Sánchez-de-Pablo (2015) explican que, en general, el diseño y la implementación de prácticas de GC son una tarea difícil para los administradores, pues la eficacia y el éxito de este tipo de prácticas dependen, en gran medida, de su adaptación óptima a los factores organizativos. En consecuencia, los gerentes deben establecer en el contexto condiciones ideales para impulsar y optimizar el uso en la organización de las prácticas de GC, y las iniciativas mediante el diseño de instrumentos, tales como las prácticas con la gestión del talento humano, los ajustes a la cultura empresarial, la puesta en práctica de sistemas de tecnología y el establecimiento de estructuras organizacionales para la GC.

En el marco de las buenas prácticas para la GC, autores como Giles y Cormican (2014) explican que las mejores prácticas generan un mejor rendimiento de las empresas, y esto lo fundamentan en que las mejores compañías tuvieron éxito mediante la integración de varios factores al mismo tiempo y con mayor eficacia, considerando criterios de prácticas relacionadas con la estrategia, el proceso, los recursos y el clima organizacional.

En este sentido, Ou, Davison y Wong (2016), y Baxter (2015) concluyen que la existencia de sistemas de intercambio de conocimientos interactivos, también conocidos como sistemas de gestión del conocimiento conversacionales, refleja la cantidad de la creación de conocimiento y la participación de las personas en preguntas y respuestas (foro de discusión), la edición colaborativa (wikis) o por medio de un proceso de narración (weblogs).

Vasudevan y Chawan (2014) explican, igualmente, que en las Pymes el conocimiento puede venir de los propietarios, gerentes de producción, supervisores, trabajadores, proveedores, clientes, consultores, conferencias, medios de comunicación 
social, la orientación al mercado, portales y foros de conocimiento, libros, estudios de casos y documentos de investigación, entre otros.

Rossi, Kerga, Taisch y Terzi (2014) concluyen en su estudio que varias de las mejores prácticas se pueden agrupar desde la estrategia, la investigación, la comercialización, el clima y la cultura organizacional, las métricas y medición del rendimiento, la gestión de cartera, los estudios de mercado, las personas, los procesos y la tecnología. Además, agrupan en dimensiones las prácticas, desde la dimensión organizacional, la cual se refiere a todas las personas que participan en actividades de la empresa todos los días, desde la división del trabajo y de las tareas (organización del trabajo), la coordinación de las personas y actividades, los roles de los empleados, las habilidades y los conocimientos. La dimensión proceso se refiere a reglas de diseño y metodologías, el control de mecanismos y mejora del proceso, las decisiones de gestión, los factores para la toma de decisiones y las actividades de valor. La dimensión de GC está relacionada con crear, compartir, representar y reusar el conocimiento tácito y explícito.

Otra práctica importante es mencionada por Mládkováa (2015), la cual se relaciona con la comunidad de práctica, entendida como un grupo de personas que tienen algún interés común y que son capaces de compartir conocimientos, experiencias, herramientas y mejores prácticas para resolver problemas. Las relaciones humanas en una comunidad son amables y solidarias. Las organizaciones suelen beneficiarse de las comunidades de práctica en muchas direcciones; éstas comunidades aceleran la colaboración, aumentan la innovación, aumentan la velocidad y la calidad de la toma de decisiones, mejoran la organización, el aprendizaje y el rendimiento, la flexibilidad, permiten un mejor trabajo con el conocimiento y aumentan las capacidades para la transferencia de conocimiento, la colaboración, el ambiente agradable, la estabilidad, y la sensación de ser parte de algo.

Ahora bien, relacionado con las prácticas, Oyemomi, Liu, Neaga y Alkhuraiji (2016) explican que el intercambio de conocimientos es un proceso continuo e interactivo que facilita la transferencia de conocimiento tácito de los empleados, a través de procesos de comunicación efectiva, mediante el uso de un canal para la adquisición de nuevas experiencias en el contexto de conocimiento, una nueva vista de un proceso y el descubrimiento de conocimiento. Esto se apoya en lo planteado por Reed, Stringer, Fazey, Evely y Kruijsen (2014), quienes explican que los empleados pueden escuchar efectivamente, compartir conocimientos y habilidades, explorar nuevas ideas, aprender, adaptar y aplicar los conocimientos adquiridos, desde una cultura de GC. 


\section{PYMES COLOMBIA}

En Colombia, según la Ley 905 de 2004, se clasifica la pequeña y mediana empresa, Pyme, según sus activos totales y el número de empleados, tal como se puede observar en la Tabla 1.

Tabla 1. Clasificación de las Pymes.

\section{Tipo de empresa}

\begin{tabular}{lll}
\hline Mediana & $51-200$ & $5001-15000$ \\
\hline Pequeña & $11-50$ & $501-5000$ \\
Microempresa & Hasta 10 & Inferior a 500 \\
\hline
\end{tabular}

\section{Planta de personal Activos totales en salarios} mínimos mensuales vigentes

Fuente: Tomado de Bancoldex (2016).

Ahora bien, en diversas publicaciones e informes nacionales de orden académico, económico y político, se establece que en la estructura empresarial del país existe entre un $92 \%$ y un $97 \%$ de compañías que son Pymes, mientras que entre un 3\% y un $8 \%$ son empresas grandes.

\section{MÉTODOS}

Para el caso del estudio de esta investigación, se empleó un método de investigación cualitativa y cuantitativo, tipo de estudio descriptivo, explicativo y correlacional, el cual, según Mejía (2004), busca comprender por medio de significados y desde una perspectiva holística, para entender el conjunto de cualidades interrelacionadas que caracterizan a un determinado fenómeno.

\section{Muestreo}

Se aplicó una encuesta a 321 Pymes de las ciudades de Armenia, Barranquilla, Bogotá, Bucaramanga, Cali, Manizales, Medellín y Pereira, en Colombia.

Para la estructuración del cuestionario se formularon preguntas en escala Likert, las cuales se calificaron de 1 a 5, donde 1 significa que se está en desacuerdo o no realizado; 2, realizado parcialmente; 3 , realizado en intervalos; 4, realizado con regularidad; y 5 , realizado completamente. Se utilizaron pruebas de fiabilidad, validez y contenido.

\section{Categorías evaluadas}

De acuerdo a las necesidades de evaluación, se agruparon las preguntas en las siguientes categorías y variables, tal y como se puede observar en la Tabla 2: 
Tabla 2. Categorías y variables.

Categorías

\begin{tabular}{ll}
\hline Buenas prácticas & $\begin{array}{l}\text { Identificación, generación, compartir, retención y aplicación de } \\
\text { conocimiento. }\end{array}$ \\
\hline $\begin{array}{l}\text { Competencias de } \\
\text { los individuos }\end{array}$ & $\begin{array}{l}\text { Gestión de la información y la documentación, gestión de la } \\
\text { comunicación, diseño de herramientas digitales, gestión de la innovación } \\
\text { y el cambio, y gestión del aprendizaje organizacional. }\end{array}$ \\
\hline Procesos & $\begin{array}{l}\text { Consideraciones generales, procesos para identificar, generar, retener, } \\
\text { compartir y aplicar conocimiento. }\end{array}$ \\
\hline Rasgos culturales & $\begin{array}{l}\text { Tipos de organización, capital intelectual, estímulos, direccionamiento } \\
\text { estratégico y apropiación. }\end{array}$ \\
\hline TIC & $\begin{array}{l}\text { Web social y semántica para identificar, generar, retener, compartir y } \\
\text { aplicar conocimiento; TIC para el modelo SECI (socializar, exteriorizar, } \\
\text { combinar e interiorizar conocimiento) de Nonaka y Takeuchi (1999); TIC } \\
\text { para un modelo holístico de gestión de conocimiento. }\end{array}$
\end{tabular}

Fuente: Autores.

Para cada una de estas categorías se utilizaron ítems relacionados, para un total de 217 preguntas y, en este caso, se presentan los resultados concernientes a las buenas prácticas y los rasgos culturales para la gestión del conocimiento.

\section{Análisis de los datos}

Desde los planteamientos de Hair, Anderson, Tatham y Black (2007), se considera el análisis de correlación, el cual implica correlacionar, simultáneamente, varias variables métricas dependientes y otras independientes, con valores ideales por encima de 0,5 . Se determinaron los supuestos estadísticos de normalidad, heterocedasticidad y linealidad.

\section{$\underline{\text { Resultados y discusión }}$}

El desarrollo y uso de las buenas prácticas para la GC en una Pyme pasa por muchos estados, ideas, formas, estructuras y definiciones. Para el caso de este estudio, se planteó evaluar el estado de dichas prácticas para la identificación, la generación, el compartir, la retención y aplicación de conocimiento, los resultados de la evaluación se pueden observar en la Figura 1: 
Figura 1. Buenas prácticas de GC en Pymes.

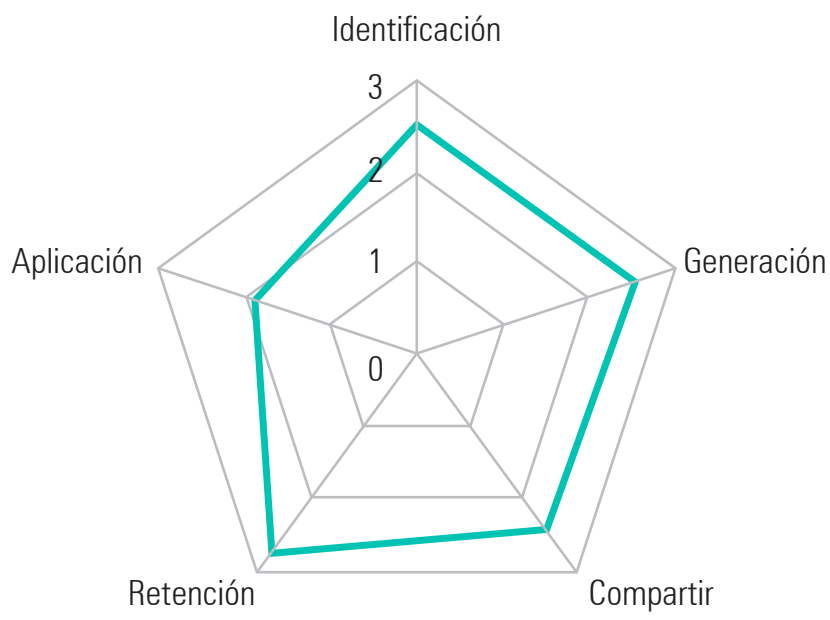

Fuente: Autores

Inicialmente, se puede observar que la valoración está por debajo de 3, lo que muestra un número bajo para las buenas prácticas; el menor valor se encontró en la Aplicación, situación preocupante, ya que más allá de los conceptos y las ideas que se pueden querer en las Pymes, los resultados muestran pobreza en esta variable, lo cual amerita la generación de acciones innovadoras que permitan la participación constante de los actores organizacionales en su quehacer y experiencia, articulados con los de sus compañeros. Le siguen Identificación, Generación, Compartir y Retención de conocimiento, con valores por debajo de 3, en una escala de 1 a 5, lo que también muestra que falta un gran camino por recorrer para hacer efectivas unas buenas prácticas de GC.

Ahora bien, siendo conocedores de la importancia de la cultura organizacional y su papel frente al logro de los objetivos de una Pyme, desde cada una de las tareas y actividades que pueden desarrollar los empleados y las dinámicas propias que deben diseñarse en el marco de una estructura apropiada, para que la GC sea una realidad y un proceso continuo de vida organizacional, los resultados de la evaluación se pueden observar en la Figura 2. 
Figura 2. Cultura de GC en Pymes.

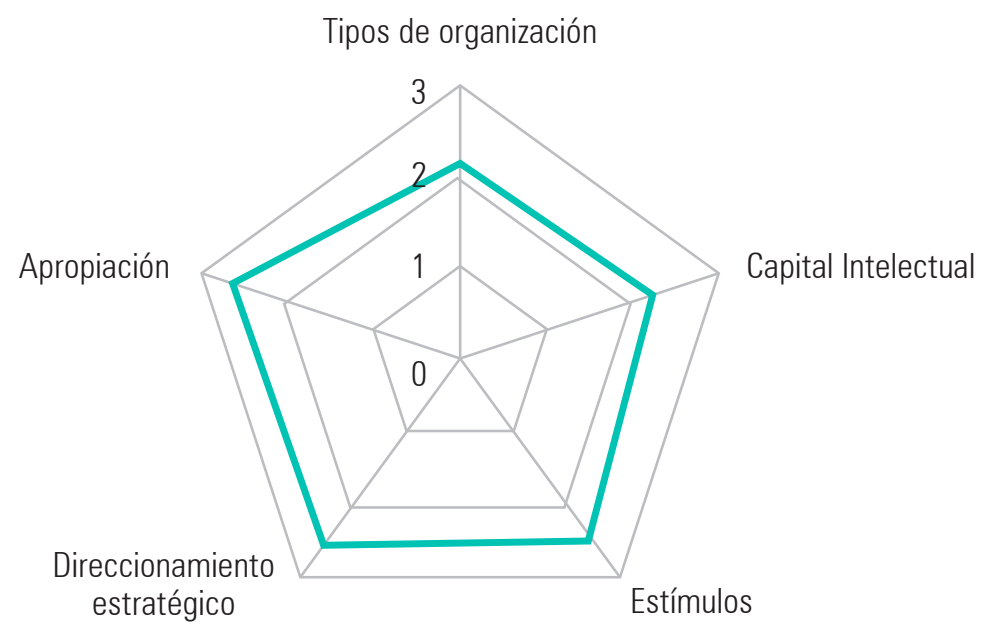

Fuente: Autores

Como una tendencia de la evaluación, se puede observar que la valoración está por debajo de 3, lo que muestra un número bajo para la cultura organizacional para la GC; el menor valor se encontró en el Tipo de Organización, relacionado con si la consideración de la clase de compañía afecta la cultura para la GC y parece que es así, pues los resultados muestran pobreza en esta variable y es, precisamente, fundamento para diseñar estrategias investigativas que permitan profundizar y generar conocimiento que supere tales falencias. Le siguen Capital Intelectual, Estímulos, Direccionamiento Estratégico y Apropiación del conocimiento, con valores por debajo de 3, en una escala de 1 a 5, lo que también muestra que falta un gran camino por recorrer para una cultura organizacional para la GC.

Se destaca una variable valorada por debajo de 2 y es la de Estímulos, lo que demuestra que los empresarios poco están haciendo por la motivación de sus empleados para hacer realidad la GC y esto corrobora la percepción que se tiene en este tipo de compañías frente a la limitación de recursos o los estilos de micro-gerencia absolutamente enfocados a los costos.

Con estos resultados se vio la necesidad de determinar la relación existente entre las buenas prácticas de GC y la cultura organizacional desde: identificación (BPIDENCON), generación (BPGENCON), compartir (BPCOMPCON), retención (BPRETCON), aplicación (BPAPLCON), tipo de organización (RSGTIPORG), capital intelectual (RSGCI), estímulos (RSGEST), direccionamiento estratégico (RSGDIREST) y apropiación (RSGAPRCON) del conocimiento, considerando los análisis estadísticos correspondientes, de los cuales se hallaron los resultados en la Tabla 3. 
Tabla 3. Correlación.

\begin{tabular}{|c|c|c|c|c|c|c|c|c|c|c|}
\hline BPIDENCON & 1 & & & & & & & & & \\
\hline BPGENCON & ,771 & 1 & & & & & & & & \\
\hline BPCOMPCON & 672 & 677 & 1 & & & & & & & \\
\hline BPRETCON &, 544 &, 571 &, 609 & 1 & & & & & & \\
\hline BPAPLCON &, 589 & ,609 & ,626 &, 593 & 1 & & & & & \\
\hline RSGTIPORG &, 550 &, 515 & ,464 & ,478 & ,487 & 1 & & & & \\
\hline RSGCl &, 529 &, 490 &, 423 & ,396 &, 433 &, 856 & 1 & & & \\
\hline RSGEST &, 595 &, 543 & ,477 & ,403 &, 406 & 612 &, 602 & 1 & & \\
\hline RSGDIREST &, 585 &, 579 &, 514 & ,428 & ,427 & ,647 & ,642 & ,701 & 1 & \\
\hline RSGAPRCON &, 581 &, 546 &, 470 & ,459 & ,422 &, 569 &, 559 & ,743 &, 736 & 1 \\
\hline
\end{tabular}

Fuente: Autores.

Como se observa en la tabla anterior, más del 30\% de los indicadores se encuentran dentro de los límites recomendados (mayores a o,5), lo cual expresa que hay una relación directa entre las variables mencionadas. Los resultados obtenidos muestran que las buenas prácticas para la GC están directamente relacionadas con la cultura organizacional para la GC.

Estos resultados están en la misma línea de los obtenidos por Le-Nguyen, Harindranath y Dyerson (2014), quienes demuestran en su estudio la interacción entre las personas y los procesos de GC; o los presentados por Hun, Lee, Chun y Benbasat (2014), quienes sugieren que para todas las empresas, en todas las circunstancias, existe un único enfoque para la GC adoptado por estas. De acuerdo con ello, las mejores prácticas y la cultura afectan positivamente los resultados de la empresa.

Igualmente, Ou, Davison y Wong (2016) concluyen que tanto las preferencias individuales, como los aspectos culturales influyen en las prácticas de GC. De igual manera, Giles y Cormican (2014)encontraron en su investigación que existe una relación entre la estrategia, los recursos, los procesos y el clima organizacional para la GC; al igual que Murphy y Coffey (2013), quienes explican que, en el marco de la cultura organizacional, la posibilidad de los empleados de compartir conocimiento genera una influencia importante en el comportamiento individual y grupal, por la forma en que esto cambia las conductas y habilidades en las interacciones entre personas. También Spraggon y Bodolica (2012) hallaron que la cultura, la estrategia de negocios y las TIC se encuentran entre los factores más importantes que facilitan la transferencia de conocimiento. 


\section{CONCLUSIONES}

La cultura organizacional influye de forma positiva y significativa en el impacto que tienen las buenas prácticas para la gestión del conocimiento y esto debido a los esfuerzos que vienen haciendo las compañías en el reconocimiento de la importancia de la GC. Esto responde a la pregunta de investigación, cumpliendo los objetivos propuestos.

El trabajo que hagan las empresas para generar mayores niveles de compromiso por parte de los empleados, para hacer realidad la GC, tiene que ver también con la posibilidad de ofrecer incentivos de diversos tipos para el bienestar de los mismos.

Se nota, desde el direccionamiento estratégico, que se ha avanzado poco en la definición de estrategias que permitan que las compañías se enfoquen en hacer realidad la GC desde los planteamientos y, por supuesto, desde la aplicación.

El estudio se limitó por la falta de respuesta de las personas del ámbito empresarial, lo que hace pensar en la necesidad de generar espacios de reconocimiento sobre la importancia de participar en este tipo de estudios.

\section{AGRADECIMIENTOS}

Agradecemos a la Universidad de Caldas, UNICALDAS, Facultad de Ingeniería, Departamento de Sistemas e Informática, grupo de investigación en Tecnologías de información y Redes GITIR, por el apoyo en la realización de esta investigación.

\section{REFERENCIAS}

Alavi, M., \& Leidner, D. E. (2001). Review: Knowledge Management and Knowledge Management Systems: Conceptuals Foundations and Research Issues. MIS Quarterly, 25(1), 107-136.

Allameha, M., Zamani, M., \& Reza-Davoodia, S. M. (2011). The Relationship between Organizational Culture and Knowledge Management. Procedia Computer Science, 3, 1224-1236.

Andreu, R., \& Sieber, S. (1999). La gestión integral del conocimiento y del aprendizaje. Economía Industrial, 326, 63-72.

Bacoldex (2016). Clasificación de empresas en Colombia. Recuperado de https:/www.bancoldex.com/Sobremicroempresas/Clasificacion-de-empresas-en-Colombia315.aspx

Barney, J. (1991). Firm Resources and Sustained Competitive Advantage. Journal de of management, 17(1), 99-120.

Baxter, H. (2015). Specialized knowledge transfer: Accelerating the expertise development cycle. Procedia Manufacturing, 3, 1465-1472.

Collins, C. J., \& Smith, K. G. (2006). Knowledge exchange and combination: The role of human resource practices in the performance of high-technology firms. Academy of Management Journal, 49(3), 544-560. 
Donate, M., \& Sánchez-de-Pablo, J. (2015). The role of knowledge-oriented leadership in knowledge management practices and innovation. Journal of Business Research, 68, 360-370.

Dosi, G., Teece, D., \& Winter, S. (1991). Toward a theory of corporate coherence: preliminary remarks. En G. Dosi, R. Giannetti \& P. A. Toninelli (eds.), Technology and Enterprise in a Historical Perspective. Oxford: Oxford University Press.

Durana, C., Çetindereb, A., \& Şahanc, Ö. (2014). An analysis on the relationship between total quality management practices and knowledge management: The case of Eskișehir. Procedia - Social and Behavioral Sciences, 109, 65-77.

Giles, T., \& Cormican, K. (2014). An empirical analysis of best management practices at the front end of the innovation process in the medical technology industry. Procedia Technology, 16, 913-920.

Hair, J., Anderson, R., Tatham, R. \& Black, W. (2007). Análisis multivariante. Madrid: Pearson

Hun, K.-T., Lee, J.-N., Chun, J.-U., \& Benbasat, I. (2014). Understanding the effect of knowledge management strategies on knowledge management performance: A contingency perspective. Information \& Management, 51, 398-416.

Le-Nguyen, F., Harindranath, G., \& Dyerson, R. (2014). Understanding knowledge management softwareorganisationmisalignments from an institutional perspective: A case study of a global IT-management consultancy firm. International Journal of Information Management, 34, 226-247.

Li, S.-T. \& Tsai, M.-H. (2009). A dynamic taxonomy for managing knowledge assets. Technovation, 29, 284-298.

Lin, Y.-C., \& Lee, H.-Y. (2012). Developing project communities of practice-based knowledge management system in construction. Automation in Construction, 22, 422-432.

Mejía Navarrete., J. (2004). Sobre la investigación cualitativa. Nuevos conceptos y campos de desarrollo. Investigaciones Sociales, 8(13), 277-299.

Mesmer-Magnus, J. R., \& DeChurch, L. A. (2009). Information sharing and team performance: A meta-analysis. Journal of Applied Psychology, 94(2), 535-546.

Mládkováa, L. (2015). Dysfunctional Communities of Practice-Thread for Organization. Procedia - Social and Behavioral Sciences, 210, 440-448.

Murphy, G., \& Coffey, V. (2013). Organizational culture and willingness to share knowledge: A competing values perspective in Australian context. International Journal of Project Management, 31(8), 1163-1174. 
Nonaka, I., \& Takeuchi, H. (1999). La organización creadora de conocimiento. México D. F.: Oxford University Press.

Ou, C., Davison, R., \& Wong, L. (2016). Using interactive systems for knowledge sharing: The impact of individual contextual preferences in China. Information \& Management, 53, 145-156.

Oyemomi, O., Liu, S., Neaga, I., \& Alkhuraiii, A. (2016). How knowledge sharing and business process contribute to organizational performance: Using the fsQCA approach. Journal of Business Research, 69(11), 52225227.

Power, R., \& Cormicana, K. (2015). Towards effective knowledge transfer in high-tech project environments: Preliminary development of key determinants. Procedia Computer Science, 64, 17-23.

Reed, M., Stringer, L., Fazey, I., Evely, A., \& Kruijsen, J. (2014). Five principles for the practice of knowledge exchange in environmental management. Journal of Environmental Management, 146, 337-345.

Riesco, M. (2006). El negocio es el conocimiento. Madrid: Díaz de Santos.

Rossi, M., Kerga, E., Taisch, M., \& Terzi, S. (2014). Engineering and Design Best Practices in New Product Development: an Empirical Research. Procedia CIRP, 21, 455-460.

Spraggon, M., \& Bodolica, V. (2012). A multidimensional taxonomy of intra-firm knowledge transfer processes. Journal of Business Research, 65, 1273-1282.

Terzieva, M. (2014). Project Knowledge Management: how organizations learn from experience. Procedia Technology, 16, 1086-1095.

Vasudevan, H., \& Chawan, A. (2014). Demystifying Knowledge Management in Indian Manufacturing SMEs. Procedia Engineering, 97, 1724-1734.

Villar, C., Alegre, J., \& Pla-Barber, J. (2014). Exploring the role of knowledge management practices on exports: A dynamic capabilities view. International Business Review, 23, 38-44.

Wang, M.-H., \& Yang, T.-Y. (2016). Investigating the success of knowledge management: An empirical study of small and medium-sized enterprises. Asia Pacific Management Review, 21, 79-91.

Wiewiora, A., Trigunarsyah, B., Murphy, G., \& Coffey, V. (2013). Organizational culture and willingness to share knowledge: A competing values perspective in Australian context. International Journal of Project Management, 31, 1163-1174. 